\title{
Automated Pipeline for Training Dataset Creation from Unlabeled Audios for Automatic Speech Recognition (Conference Paper)
}

\author{
Romanovskyi, O.a, Iosifov, I.a, Iosifova, O.a, Sokolov, V.b, Kipchuk, F.b, Sukaylo, I. ${ }^{\mathrm{b}}$ \\ aUkraine Ender Turing OÜ, Tallinn, Estonia \\ 'Borys Grinchenko Kyiv University, Kiev, Ukraine
}

\begin{abstract}
In the paper, we present a software pipeline for speech recognition to automate the creation of training datasets, based on desired unlabeled audios, for low resource languages and domain-specific area. Considering the commoditizing of speech recognition, more teams build domain-specific models as well as models for local languages. At the same time, lack of training datasets for low to middle resource languages significantly decreases possibilities to exploit last achievements and frameworks in the Speech Recognition area and limits the wide range of software engineers to work on speech recognition problems. This problem is even more critical for domain-specific datasets. The pipeline was tested for building Ukrainian language recognition and confirmed that the created design is adaptable to different data source formats and expandable to integrate with existing frameworks. (C) 2021, The Author(s), under exclusive license to Springer Nature Switzerland AG.
\end{abstract}

\section{Author keywords}

ASR; asynchronous graphs; automatic speech recognition; dataset creation pipeline; natural language processing; NLP

\section{Funding details}

Funding sponsor Funding number Acronym

Ministry of Education - Singapore CCNU19TSO22 MOE

\section{Funding text}

This scientific work was partially supported by RAMECS and self-determined research funds of CCNU from the colleges' primary research and operation of MOE (CCNU19TSo22). The research team is grateful to Ender Turing OÜ for defining the business problem, comments, corrections, inspiration, and computational.

\section{About this paper}

https://link.springer.com/chapter/10.1007/978-3-030-80472-5 3

ISSN: 2194-5365

Print ISBN: $978-3-030-80472-5$

DOI: $10.1007 / 978-3-030-80472-5 \quad 3$

EID: 2-S2.0-85111941280
Source Type: Book Series Document Type: Conference Paper Publisher: Springer, Cham 